\title{
Exact periodic and solitonic states of the spinor condensates in a uniform external potential
}

\author{
Zhi-Hai Zhang ${ }^{a}$, Shi-Jie Yang ${ }^{b *}$ \\ ${ }^{a}$ School of Physics and Electronics, Yancheng Teachers University, Yancheng, 224051, \\ China \\ ${ }^{b}$ Department of Physics, Beijing Normal University, Beijing 100875, China
}

\begin{abstract}
We propose a method to analytically solve the one-dimensional coupled nonlinear Gross-Pitaevskii equations which govern the motion of the spinor BoseEinstein condensates. In a uniform external potential, several classes of exact periodic and solitonic solutions, either in real or in complex forms, are obtained for both the $F=1$ and $F=2$ condensates for the Hamiltonian comprising the kinetic energy, the linear and the quadratic Zeeman energies. Real solutions take the form of composite soliton trains. Complex solutions correspond to the mass counter-flows as well as spin currents. These solutions are general that contains neither approximations nor constraints on the system parameters.
\end{abstract}

Keywords: Exact solution; Spinor condensate; Coupled GP equations.

\section{Introduction}

The experimental achievement and rapid development of Bose-Einstein condenstates(BECs) of alkaline atoms[1], triggered the study of atomic BECs, which takes place in the systems of bosonic atoms at ultralow temperatures, as well as in optical wave systems. One of the particularly interesting directions in that regard is the study of multicomponent BEC systems and solitary waves in them, which constitute a platform that is ideal for the study of numerous nonlinear wave phenomena $[2,3,4]$. This is a subject of broad interest,

\footnotetext{
*Corresponding author

Email address: yangshijie@tsinghua.org.cn (Shi-Jie Yang ${ }^{b}$ )
}

Preprint submitted to elsevier

May 8, 2016

(C) 2016. This manuscript version is made available under the Elsevier user license http://www.elsevier.com/open-access/userlicense/1.0/ 
not only in the realm of atomic BECs[5, 6], but also in plasma physics[7], hydrodynamics[8], nonlinear optics[9], and some organic materials[10]. In the mean-field approximation, the BEC dynamics at ultra-low temperatures is accurately described by Gross-Pitaevskii equations (GPEs). A counterpart of the GPEs, which is a basic model in nonlinear optics, is the nonlinear Schrödinger equation. Therefore, the study of GPEs is primordial importance, which is a very valuable tool to deepen our understanding of condensed matter related issues.

Recently, many studies have been performed either by solving the corresponding GPE numerically $[11,12,13]$ or by using perturbation methods. However, the construction of exact solutions of the GPE can help to advance our understanding of various physical phenomena governed by this nonlinear equation. For instance, the exact analytical solutions can contribute to select the experimental parameters, to analyze the stability of BECs and to check numerical analysis of this nonlinear equation. D. Carr et al. have presently a new family of solutions of a repulsive in a quasi-1D geometry, where the potential itself was fix to be a Jacobi elliptic function. R.A.Van Gorder et al. obtained stationary solutions for the $1+1$ nonlinear Schrödinger equation with a free potential and small yet arbitrary potential, which can be constructed by assuming a perturbation solution in the Jacobi elliptic function solution. These works laid the foundation for our present work $[14,15,16]$. Analytically, some solitonic solutions are obtained by means of variable functions or similar transformation for time or spatial modulated coupling constants[17, 18, 19, 20]. Various approximations are employed to study the solitons such as bright and dark solitons in the $F=1$ spinor BECs[21, 22, 23, 24]. Exact solutions are usually difficult to obtain due to the complexity of the coupled nonlinear GPEs. The challenges are two-fold: one is the nonlinear density-density interactions, while the other is the spin-exchange couplings between the hyperfine states. In our previous publications we have given exact solutions for the $F=1$ and $F=2$ spinor BECs for some special cases[25, 26]. In this paper, we propose a general method which simultaneously decouples the nonlinear density-density interactions and the spin-spin interactions in the GPEs. Classes of the exact solutions, either in real or in complex forms, are systematically constructed for the Hamiltonian containing the kinetic energy, the linear and the quadratic Zeeman energies. The solutions are expressed by combinations of the Jacobi elliptic functions for periodic solutions or the hyperbolic functions for solitonic solutions. The composite solitons are identified as vector solitons or 
scalar solitons, respectively.

The paper is organized as follows: In Sec.II we described the method and systematically present solutions for the spin-1 condensates. In Sec.III we give a solution to the spin-2 condensates as an example. Section IV contains a brief summary.

\section{2. spin-1 condensates}

In a uniform external potential $(V(\mathbf{r})=0)$, we are concerned with the quasi-one-dimensional (1D) spinor system, which the transverse dimensions of the $\mathrm{BEC}$ are on the order of healing length, and the longitudinal dimension of the BEC is much longer than the transverse ones. In this section, we deal with the $F=1$ condensates in which the mean-field order parameters are described by a macroscopic wavefunction with three hyperfine states $\Psi=$ $\left(\psi_{+1}, \psi_{0}, \psi_{-1}\right)^{T}$. The Hamiltonian that contains the linear and the quadratic Zeeman effects reads[? ?

$H=\int d \mathbf{r}\left\{\sum_{m=-1}^{1} \psi_{m}^{*}\left[-\frac{\hbar^{2}}{2 M} \nabla^{2}+V(\mathbf{r})-p m+q m^{2}\right] \psi_{m}+\frac{\bar{c}_{0}}{2} n_{t o t}^{2}+\frac{\bar{c}_{2}}{2}|\mathbf{F}|^{2}\right\}$

where the spin-polarization vector $\mathbf{F}=\psi_{m}^{*} \hat{\mathbf{F}}^{i}{ }_{m n} \psi_{n}$ with $\hat{\mathbf{F}}^{i}(i=x, y, z)$ the spin matrices. The terms with coefficients $c_{0}$ and $c_{2}$ describe respectively the spin-independent and the spin-dependent binary elastic collisions in the combined symmetric channels of total spin 0 and 2. They are expressed in terms of the $s$-wave scattering lengths $a_{0}$ and $a_{2}$ as: $\bar{c}_{0}=4 \pi \hbar^{2}\left(a_{0}+2 a_{2}\right) / 3 M$ and $\bar{c}_{2}=4 \pi \hbar^{2}\left(a_{2}-a_{0}\right) / 3 M . \quad p$ and $q$ are linear and quadratic Zeeman coupling coefficients. $n_{\text {tot }}=\left|\psi_{1}\right|^{2}+\left.\psi_{0}\right|^{2}+\left|\psi_{-1}\right|^{2}$ and $V(\mathbf{r})$ is the external potential.

The dynamical motion of the $F=1$ spinor wavefunctions are governed by $i \partial_{t} \psi_{m}=\delta H / \delta \psi_{m}^{*}$, which are explicitly written as the coupled nonlinear GPEs,

$$
\begin{aligned}
i \partial_{t} \psi_{m} & =\left[-\frac{\hbar^{2}}{2 M} \partial_{x}^{2}-p m+q m^{2}+c_{0} n_{t o t}\right] \psi_{m} \\
& +c_{2} \sum_{n=-1}^{1} \mathbf{F} \cdot \hat{\mathbf{F}}^{i} \psi_{n},(m=1,0,-1)
\end{aligned}
$$

where $c_{0}=\bar{c}_{0} / 2 a_{\perp}^{2}$ and $c_{2}=\bar{c}_{2} / 2 a_{\perp}^{2}$ are the reduced coupling constants with $a_{\perp}$ the transverse width of the quasi-1D system. 
Below we choose discussions $\hbar=M=1$ as the units for convenience. By substituting the wavefunction $\Psi(x, t)$ with

$$
\left(\begin{array}{c}
\psi_{1}(x, t) \\
\psi_{0}(x, t) \\
\psi_{-1}(x, t)
\end{array}\right) \rightarrow\left(\begin{array}{c}
\psi_{1}(x) e^{-i\left(\mu+\mu_{1}\right) t} \\
\psi_{0}(x) e^{-i \mu t} \\
\psi_{-1}(x) e^{-i\left(\mu-\mu_{1}\right) t}
\end{array}\right)
$$

we obtain the stationary GPEs as,

$$
\begin{aligned}
\left(\mu+\mu_{1}\right) \psi_{1} & =\left[-\frac{1}{2} \partial_{x}^{2}+\left(c_{0}+c_{2}\right)\left(\left|\psi_{1}\right|^{2}+\left|\psi_{0}\right|^{2}\right)+\left(c_{0}-c_{2}\right)\left|\psi_{-1}\right|^{2}\right. \\
& -p+q] \psi_{1}+c_{2} \psi_{0}^{2} \psi_{-1}^{*}, \\
\mu \psi_{0} & =\left[-\frac{1}{2} \partial_{x}^{2}+\left(c_{0}+c_{2}\right)\left(\left|\psi_{1}\right|^{2}+\left|\psi_{-1}\right|^{2}\right)+c_{0}\left|\psi_{0}\right|^{2}\right] \psi_{0} \\
& +2 c_{2} \psi_{0}^{*} \psi_{1} \psi_{-1}, \\
\left(\mu-\mu_{1}\right) \psi_{-1} & =\left[-\frac{1}{2} \partial_{x}^{2}+\left(c_{0}+c_{2}\right)\left(\left|\psi_{-1}\right|^{2}+\left|\psi_{0}\right|^{2}\right)+\left(c_{0}-c_{2}\right)\left|\psi_{1}\right|^{2}\right. \\
& +p+q] \psi_{-1}+c_{2} \psi_{0}^{2} \psi_{1}^{*} .
\end{aligned}
$$

Since the chemical potential of each hyperfine state is different, Eqs. (4) are not really stationary equations, given $\mu_{1} \neq 0$. It comprises a "Lamor precession" between the hyperfine states. However, the density profile of each state are time-invariant so we simply call them the stationary states.

The periodic boundary conditions,

$$
\psi_{m}(1)=\psi_{m}(0), \quad \psi_{m}^{\prime}(1)=\psi_{m}^{\prime}(0)
$$

is adopted in our calculations. In the following, we consider several types of real and complex solutions for $F=1$ condensates.

In order to seek the analytical solutions we decouple spin-spin interactions in the Eqs.(4) by assuming $\psi_{-m}^{*}= \pm \psi_{m}$. It is directly to check that $\psi_{-m}^{*}=$ $\psi_{m}$ corresponds to (partially) spin-polarized states $(|\mathbf{F}| \neq 0)$ whereas $\psi_{-m}^{*}=$ $-\psi_{m}$ corresponds to spin-unpolarized or polar states $(|\mathbf{F}|=0)$. On the other hand, the nonlinear density-density couplings between the hyperfine states are decoupled by making use of the properties of the Jacobi elliptic functions or the hyperbolic functions. There are real and complex forms of solutions which are explicitly described as follows. 


\subsection{Real solutions}

We present a brief derivation in which we require that the wave function is approximately separable, and that its transverse parts are not excited. By requiring the transverse dimensions to be on the order of the healing length. Therefore, in a uniform external potential we consider the following sn-cn-sn form of solutions to the nonlinear Eq.(4),

$$
\left(\begin{array}{l}
\psi_{1}(x) \\
\psi_{0}(x) \\
\psi_{-1}(x)
\end{array}\right)=\left(\begin{array}{l}
A \operatorname{sn}(k x, m) \\
D \operatorname{cn}(k x, m) \\
-A \operatorname{sn}(k x, m)
\end{array}\right),
$$

where sn and $c n$ are the Jacobi elliptic functions of modulus $m$ with period $k=4 j K(m)$ ( $j$ is an integer and are set $j=2$ in this paper), where $K(m)$ is an elliptic integral of the first kind. Thus the periodic boundary conditions (Eq.(5)) are satisfied if $k=4 j K(m)$, where $j \in\{1,2,3, \ldots\}$. The number of nodes in the $j$ th solution is $2 j$. $A$ and $D$ are the real amplitudes. With Jacobian elliptic identities $\mathrm{sn}^{2}+\mathrm{cn}^{2}=1$. One has

$$
\left|\psi_{-1}\right|^{2}=\left|\psi_{1}\right|^{2}, \quad\left|\psi_{0}\right|^{2}=D^{2}-\frac{D^{2}}{A^{2}}\left|\psi_{1}\right|^{2}
$$

Substituting (7) into the equations (4), we obtain three decoupled equations

$$
\tilde{\mu}_{m} \psi_{m}=-\frac{1}{2} \psi_{m}^{\prime \prime}+\tilde{\gamma}_{m}\left|\psi_{m}\right|^{2} \psi_{m}, \quad(m=0, \pm 1)
$$

where the effective chemical potentials $\tilde{\mu}_{m}$ and interacting constants $\tilde{\gamma}_{m}$ are defined as

$$
\begin{aligned}
& \tilde{\mu}_{ \pm 1}=\mu-q-c_{0} D^{2} \\
& \tilde{\mu}_{0}=\mu-2 c_{0} A^{2} \\
& \tilde{\gamma}_{ \pm 1}=2 c_{0}-c_{0} \frac{D^{2}}{A^{2}} \\
& \tilde{\gamma}_{0}=c_{0}-2 c_{0} \frac{A^{2}}{D^{2}} .
\end{aligned}
$$

In order to obtain self-consistent solutions, one should take $p=-\mu_{1}$. Therefore, the linear Zeeman energy plays the role of balancing the chemical

potentials between the hyperfine states $\psi_{1}$ and $\psi_{-1}$. The effective chemical potentials $\tilde{\mu}_{m}$ and amplitudes $A, D$ can be obtained as,

$$
\begin{aligned}
& \tilde{\mu}_{ \pm 1}=\frac{1}{2} k^{2}\left(1+m^{2}\right) \\
& \tilde{\mu}_{0}=\frac{1}{2} k^{2}\left(1-2 m^{2}\right) \\
& A^{2}=\frac{m^{2} k^{2}}{\tilde{\gamma}_{ \pm 1}} \\
& D^{2}=-\frac{m^{2} k^{2}}{\tilde{\gamma}_{0}} .
\end{aligned}
$$


Formula (10) implies that the effective intra-species interactions for hyperfine states $\psi_{ \pm 1}$ should be repulsive $\left(\tilde{\gamma}_{ \pm 1}>0\right)$ while for $\psi_{0}$ be attractive $\left(\tilde{\gamma}_{0}<0\right)$. It imposes constraints on the relations among parameters $c_{0}, q$ and $\mu$.

Figure 1(a) illustrates the density profiles of each hyperfine states in solution (6). The parameters are chosen as $c_{0}=30, q=-13.7707, A=2.0061$, $D=2.6704, m=0.4$ and $\mu=300$. The plot demonstrates that the solutions are unique. This state has vanishing spin-polarization $|\mathbf{F}|=0$. The modulus of the Jacobi elliptic functions is a free parameter. As $m \rightarrow 1$, we naturally obtain the periodic soliton train solution. The single soliton solution can be obtained by directly substituting the Jacobi elliptic functions with the hyperbolic functions in (6). Namely,

$$
\left(\begin{array}{l}
\psi_{1}(x) \\
\psi_{0}(x) \\
\psi_{-1}(x)
\end{array}\right)=\left(\begin{array}{l}
A \tanh (k x) \\
D \operatorname{sech}(k x) \\
-A \tanh (k x)
\end{array}\right),
$$

This solution has been addressed in literatures[11, 13]. The density profile is displayed in Fig.1(b), with the parameters $c_{0}=1.3, k=2, q=-2$, $A=1.6408, D=1.5191$ and $\mu=5$. It is a dark-bright-dark composite soliton. We refer to the behavior at each node of $\Psi$ as a kink. $|\Psi|^{2}$ is proportional to the density of particles in a BEC. This density is constant everywhere except at the kink.

Other forms of real solutions can also be constructed by the same way. For example, we seek the cn-sn-cn form of solution to Eq.(4),

$$
\left(\begin{array}{l}
\psi_{1}(x) \\
\psi_{0}(x) \\
\psi_{-1}(x)
\end{array}\right)=\left(\begin{array}{l}
A \operatorname{cn}(k x, m) \\
D \operatorname{sn}(k x, m) \\
-A \operatorname{cn}(k x, m)
\end{array}\right) .
$$

One has

$$
\left|\psi_{-1}\right|^{2}=\left|\psi_{1}\right|^{2}, \quad\left|\psi_{0}\right|^{2}=D^{2}-\frac{D^{2}}{A^{2}}\left|\psi_{1}\right|^{2}
$$

Eq.(4) are again decoupled into (8). It follows that the effective chemical potentials $\tilde{\mu}_{m}$ and interacting constants $\tilde{\gamma}_{m}$ as

$$
\begin{aligned}
& \tilde{\mu}_{ \pm 1}=\mu-q-c_{0} D^{2} \\
& \tilde{\mu}_{0}=\mu-2 c_{0} A^{2} \\
& \tilde{\gamma}_{ \pm 1}=2 c_{0}-c_{0} \frac{D^{2}}{A^{2}} \\
& \tilde{\gamma}_{0}=c_{0}-2 c_{0} \frac{A^{2}}{D^{2}} .
\end{aligned}
$$


The effective chemical potentials $\tilde{\mu}_{m}$ and amplitudes $A, D$ are self-consistently calculated,

$$
\begin{aligned}
& \tilde{\mu}_{ \pm 1}=\frac{1}{2} k^{2}\left(1-2 m^{2}\right) \\
& \tilde{\mu}_{0}=\frac{1}{2} k^{2}\left(1+m^{2}\right) \\
& A^{2}=-\frac{m^{2} k^{2}}{\tilde{\gamma}^{ \pm 1}} \\
& D^{2}=\frac{m^{2} k^{2}}{\tilde{\gamma}_{0}} .
\end{aligned}
$$

Formula (15) reveal that the effective intra-species interactions for the hyperfine states $\psi_{ \pm 1}$ should be attractive $\left(\tilde{\gamma}_{ \pm 1}<0\right)$ while for $\psi_{0}$ be repulsive $\left(\tilde{\gamma}_{0}>0\right)$.

Figure 2(a) plots the density profiles of each hyperfine states (12). The parameters are $c_{0}=-10, q=22.734, A=3.9602, D=5.1788, m=0.5$ and $\mu=-200$. The spin is unpolarized $|\mathbf{F}|=0$. Similarly, the single soliton solution is constructed by substituting sn $\longrightarrow$ tanh and $\mathrm{cn} \longrightarrow$ sech. Figure 2(b) is the density profiles of a typical bright-dark-bright soliton with $c_{0}=-1, k=3, q=4.5, A=2.2913, D=1.2247$ and $\mu=-1.5$.

\subsection{Complex solutions}

We can also take complex form of periodic solutions to the nonlinear Eq.(4) as,

$$
\left(\begin{array}{l}
\psi_{1} \\
\psi_{0} \\
\psi_{-1}
\end{array}\right)=\left(\begin{array}{l}
f(x) e^{i \theta(x)} \\
D \operatorname{sn}(k x, m) \\
f(x) e^{-i \theta(x)}
\end{array}\right)
$$

where $f(x)=\sqrt{A+B \mathrm{cn}^{2}(k x, m)}$. $A, B$ and $D$ are real constants. One has

$$
\left|\psi_{-1}\right|^{2}=\left|\psi_{1}\right|^{2}, \quad\left|\psi_{0}\right|^{2}=D^{2}\left(1+\frac{A}{B}\right)-\frac{D^{2}}{B}\left|\psi_{1}\right|^{2} .
$$

Substituting (17) into the coupled GPEs (4). We again obtain the decoupled equations (8) with the effective chemical potentials and intr-species interaction constants,

$$
\begin{aligned}
& \tilde{\mu}_{ \pm 1}=\mu-q-\left(c_{0}+2 c_{2}\right) \frac{D^{2}}{B}(A+B) \\
& \tilde{\mu}_{0}=\mu-2\left(c_{0}+2 c_{2}\right)(A+B) \\
& \tilde{\gamma}_{ \pm 1}=2 c_{0}-\left(c_{0}+2 c_{2}\right) \frac{D^{2}}{B} \\
& \tilde{\gamma}_{0}=c_{0}-2\left(c_{0}+2 c_{2}\right) \frac{B}{D^{2}}
\end{aligned}
$$


To obtain the self consistent solution we should set $\mu_{1}=-p$, which yields to

$$
\begin{aligned}
& B=-\frac{m^{2} k^{2}}{\tilde{\gamma}_{1}}, \\
& A=\frac{2 \tilde{\mu}_{ \pm 1}-\left(1-2 m^{2}\right) k^{2}}{3 \tilde{\gamma}_{ \pm 1}}, \\
& D^{2}=\frac{m^{2} k^{2}}{\tilde{\gamma}_{0}} .
\end{aligned}
$$

We note that the effective interactions $\tilde{\gamma}_{0}>0$. The phase is

$$
\theta(x)=\int_{0}^{x} \frac{\alpha}{f^{2}(\xi)} d \xi
$$

where $\alpha= \pm\left(2 \tilde{\mu}_{ \pm 1} A^{2}-2 \tilde{\gamma}_{ \pm 1} A^{3}+k^{2} A B\left(1-m^{2}\right)\right)^{\frac{1}{2}}$ is an integral constant. The periodic boundary conditions (5) require that the amplitudes and phase satisfy

$$
f(1)=f(0), \quad \theta(1)-\theta(0)=2 j \pi \times n,
$$

respectively. Here $n$ is an integer. The periodic condition for the phase is satisfied by properly adjusting the modulus $m$ of the Jacobi elliptic functions. We note that all parameters are monotonic in $m$, so that our algorithm is quite straightforward. Figure 3(a) and (b) display the phase and density profiles of the complex solution (16), respectively. The parameters are taken as $n=2, c_{0}=34, c_{2}=43, q=17, A=1.524, B=-0.5795, D=$ 1.0766, $m=0.82$ and $\mu=448.6349$. As $m \rightarrow 1$, we obtain the soliton train solution. In Fig3(a), there was a background linear slope with a jump where the density notches occurred, which corresponded to the mass counter-flows as well as spin currents, yet the density distributions remain stationary. In addition, the number of the solitons corresponded to the number of the phase step [27, 28].

Similarly, the single soliton solution is obtained by substituting the Jacobi elliptic functions with the hyperbolic functions as

$$
\left(\begin{array}{l}
\psi_{1} \\
\psi_{0} \\
\psi_{-1}
\end{array}\right)=\left(\begin{array}{l}
f(x) e^{i \theta(x)} \\
D \tanh (k x) \\
f(x) e^{-i \theta(x)}
\end{array}\right)
$$

where $f(x)=\sqrt{A+B \operatorname{sech}^{2}(k x)}$. Figure 3(c) and (d) plots a typical greydark-grey soliton for the solution (22) with $n=6, c_{0}=3, c_{2}=4, q=5$, $k=8.7, A=5.2032, B=-2.7032, D=2.3252$ and $\mu=130.69$. Here the phases $\theta(x)$ should satisfy the periodic condition (5) by adjusting the 
width of the soliton $k$. Since $(|\mathbf{F}| \neq 0)$, this type of composite soliton may be properly called the polarized or vector soliton.

The spin-unpolarized $(\mathbf{F}=0)$ complex solution can be constructed in the following form,

$$
\left(\begin{array}{l}
\psi_{1} \\
\psi_{0} \\
\psi_{-1}
\end{array}\right)=\left(\begin{array}{l}
f(x) e^{i \theta(x)} \\
D \operatorname{cn}(k x, m) \\
-f(x) e^{-i \theta(x)}
\end{array}\right)
$$

where $f(x)=\sqrt{A+B \mathrm{sn}^{2}(k x, m)}$. $A, B$ and $D$ are real constants. One has

$$
\left|\psi_{-1}\right|^{2}=\left|\psi_{1}\right|^{2}, \quad\left|\psi_{0}\right|^{2}=D^{2}\left(1+\frac{A}{B}\right)-\frac{D^{2}}{B}\left|\psi_{1}\right|^{2} .
$$

The effective chemical potentials and interaction constants are,

$$
\begin{aligned}
& \tilde{\mu}_{ \pm 1}=\mu-q-c_{0} \frac{D^{2}}{B}(A+B) \\
& \tilde{\mu}_{0}=\mu-2 c_{0}(A+B) \\
& \tilde{\gamma}_{ \pm 1}=2 c_{0}-c_{0} \frac{D^{2}}{B} \\
& \tilde{\gamma}_{0}=c_{0}-2 c_{0} \frac{B}{D^{2}}
\end{aligned}
$$

One obtains

$$
\begin{aligned}
& B=\frac{m^{2} k^{2}}{\tilde{\gamma}_{1}}, \\
& A=\frac{2 \tilde{\mu}_{ \pm 1}-\left(1+m^{2}\right) k^{2}}{3 \tilde{\gamma}_{ \pm}}, \\
& D^{2}=-\frac{m^{2} k^{2}}{\tilde{\gamma}_{0}} .
\end{aligned}
$$

The effective intra-species interactions $\tilde{\gamma}_{0}<0$.

Figure 4(a) and (b) display the phase and density profiles of the solution (23). The parameters are $n=2, c_{0}=-5, q=-190, A=4.5347, B=$ $0.4455, D=2.7731 m=0.44$ and $\mu=4$. Figure $4(\mathrm{c})$ and (d) are the corresponding single soliton solution with parameters $n=2, c_{0}=-5, q=-8, k=$ $3, A=0.6781, B=0.8719, D=1.8825$ and $\mu=-20$. It is a typical greybright-grey composite soliton. In comparison to the polarized soliton (22), we may call this spin-unpolarized soliton or scalar solitons. 


\section{3. spin-2 condensates}

Our method is also applicable to the $F=2$ condensates. For illustration, we give an example in this section. By substituting the wavefunction with[26]

$$
\left(\begin{array}{c}
\psi_{2}(x, t) \\
\psi_{1}(x, t) \\
\psi_{0}(x, t) \\
\psi_{-1}(x, t) \\
\psi_{-2}(x, t)
\end{array}\right) \rightarrow\left(\begin{array}{c}
\psi_{2}(x) e^{-i\left(\mu+\mu_{2}\right) t} \\
\psi_{1}(x) e^{-i\left(\mu+\mu_{1}\right) t} \\
\psi_{0}(x) e^{-i \mu t} \\
\psi_{-1}(x) e^{-i\left(\mu-\mu_{1}\right) t} \\
\psi_{-2}(x) e^{-i\left(\mu-\mu_{2}\right) t}
\end{array}\right)
$$

we obtain the generalized stationary GPEs as,

$$
\begin{aligned}
\left(\mu \pm \mu_{2}\right) \psi_{ \pm 2} & =\left[-\frac{1}{2} \partial_{x}^{2}+c_{0} n \pm 2 c_{1} F_{z} \mp 2 p+4 q\right] \psi_{ \pm 2} \\
& +c_{1} F_{\mp} \psi_{ \pm 1}+\frac{c_{2}}{\sqrt{5}} A \psi_{\mp 2}^{*} \\
\left(\mu \pm \mu_{1}\right) \psi_{ \pm 1} & =\left[-\frac{1}{2} \partial_{x}^{2}+c_{0} n \pm c_{1} F_{z} \mp p+q\right] \psi_{ \pm 1} \\
& +c_{1}\left(\frac{\sqrt{6}}{2} F_{\mp} \psi_{0}+F_{ \pm} \psi_{ \pm 2}\right)-\frac{c_{2}}{\sqrt{5}} A \psi_{\mp 1}^{*}, \\
\mu \psi_{0} & =\left[-\frac{1}{2} \partial_{x}^{2}+c_{0} n\right] \psi_{0}+\frac{\sqrt{6}}{2} c_{1}\left(F_{+} \psi_{1}+F_{-} \psi_{-1}\right) \\
& +\frac{c_{2}}{\sqrt{5}} A \psi_{0}^{*} .
\end{aligned}
$$

We take the following form of solution,

$$
\left(\begin{array}{l}
\psi_{2} \\
\psi_{1} \\
\psi_{0} \\
\psi_{-1} \\
\psi_{-2}
\end{array}\right)=\left(\begin{array}{l}
f(x) e^{i \theta(x)} \\
C \operatorname{sn}(k x, m) \\
D \operatorname{cn}(k x, m) \\
-C \operatorname{sn}(k x, m) \\
f(x) e^{-i \theta(x)}
\end{array}\right)
$$

where $f(x)=\sqrt{A+B \mathrm{sn}^{2}(k x, m)}$. $A, B, C$ and $D$ are real constants. The equations (29) can be decoupled and self-consistently solved in the same way as for the $F=1$ condensates. And the periodical boundary conditions (5) 
require that the amplitudes and phase $\theta(x)=\int_{0}^{x} \frac{\alpha_{1}}{f^{2}(\xi)} d \xi\left(\alpha_{1}= \pm\left(2 \tilde{\mu}_{2} A^{2}-\right.\right.$ $\left.2 \tilde{\gamma}_{2} A^{3}+k^{2} A B\right)^{\frac{1}{2}}$ is the integral constant) satisfy $f(1)=f(0), \quad \theta(1)-\theta(0)=$ $2 j \pi \times n$,respectively. Here $n$ is an integer. We need the linear Zeeman energy $\mu_{1}=-p$ and $\mu_{2}=2 p$ to balance the chemical potentials. We omit the details and just the results.

Figure 5(a) and (b) display the phase and density profiles of the solution (29) for $F=2$ condensates. The parameters are $n=2, c_{0}=10, c_{2}=$ $25, q=-53.4153, m=0.7, A=1.8, B=0.6, C=2.1278, D=1.7699$ and $\mu=210$. Figure $5(\mathrm{c})$ and (d) are results for the single soliton solution which corresponding to the periodic solution (29) with $n=5, c_{0}=10, c 2=$ $20, q=-8.2012, k=4.05, A=3.9, B=1.3, C=1.4952, D=2.429$ and $\mu=200$. It exhibits a typical grey-dark-bright-dark-grey composite soliton structure[28, 29].

\section{4. summary}

In summary, we have developed a method to systematically solve the one-dimensional coupled nonlinear GPEs which govern the motion of the spinor BECs. Several classes of exact analytical solutions for the $F=1$ and $F=2$ condensates are obtained in a uniform external potential. We have obtained the real solution of the from(6) where $\psi_{ \pm 1}$ should be repulsive while for $\psi_{0}$ be attractive. The single soliton solution can be obtained by directly substituting the Jacobi elliptic functions with the hyperbolic functions(11) where $\left|\Psi^{2}\right|$ is constant everywhere except at the kink. We also construct the other forms of real solutions(12) where $\psi_{ \pm 1}$ should be attractive while for $\psi_{0}$ be repulsive. Similarly, the single soliton solution is constructed by substituting sn $\longrightarrow$ tanh and $\mathrm{cn} \longrightarrow$ sech. And the complex solutions $(16,23)$ correspond to the mass counter-flows as well as spin currents.

Obviously, we can also obtain a lot of other analytical solutions with different combinations of the Jacobi elliptic functions. We emphasize that our method is general and exact, without any approximations, and can be extended to other nonlinear systems such as the coupled nonlinear KleinGordon equations, the dynamical coupled nonlinear Schrodinger equations, the coupled KdV equations, or the circularly symmetric coupled sexticoscillators, et al. Works in this direction are in progress.

\section{Acknowledgments:}


Project supported by the National Science Fundation of China (under Grant No. 11447193), and the University National Science Foundation of Jiangsu Province of China (under Grant No. 14KJB140015).

\section{References}

[1] D. M. Stamper-Kurn, M. P. Andrews, A. P. Chikkatur, S. Inouye, H.-J. Miesner, J. Stenger, and W. Ketterle, Phys. Rev. Lett. 80, 2027 (1998).

[2] P. G. Kevrekidis, D. J. Frantzeskakis, and R. Carretero-González, Emergent Nonlinear Phenomena in Bose-Einstein Condensates: Theory and Experiment, Springer, Heidelberg, 2008.

[3] K. Manikandan, P. Murugandam, M. Senthilvelan, and M. Lakshmanan, Phys. Rev. E 93, 032213 (2016).

[4] S. Gautam, and S. K. Adhikari, Phys. Rev. A 91, 063617 (2015).

[5] L. P. Pitaevskii, and S. Stringari, Bose-Einstein Condensation, Oxford University Press, Oxford, 2003.

[6] I. Bloch, Nat. Phys. 1, 23 (2005).

[7] R. K. Dodd, J. C. Eilbeck, J. D. Gibbon, and H. C. Morris, Solitions and Nonlinear Wave Equations, Academic Press, New York, 1982.

[8] P. L. Christiansen, J. C. Eilbeck, and R. D. Parmentier, Future Directions of Nonlinear Dynamics in Physical and Biological Systems, NATO ASI, Ser. B, vol. 312, Plenum Press, New York, 2013.

[9] Y. J. Shen, Y. T. Gao, D. W. Zou, Y. H. Sun, Y, J. Feng, and L. Xue, Phys. Rev. E 89, 062915 (2014).

[10] G. Burlak, and B. A. Malomed, Phys. Rev. E 88, 062904 (2013).

[11] L. Li, Z. Li, B. A. Malomed, D. Mihalache, and W. M. Liu, Phys. Rev. A 72,033611 (2005).

[12] W. X. Zhang, Ö. E. Müstecaplioğlu, and L. You, Phys. Rev. A 75, 043601 (2007). 
[13] H. E. Nistazakis, D. J. Frantzeskakis, P. G. Kevrekidis, B. A. Malomed and R. Carretero-González, Phys. Rev. A 77,033612(2008).

[14] J. C. Bronski, L. D. Carr, B. Deconinck, and J. N. Kutz, Phys. Rev. Lett. 86, 1402 (2001).

[15] K. Mallory, and R. A. Van Gorder, Phys. Rev. E 88, 013205 (2013).

[16] K. Mallory, and R. A. Van Gorder, Phys. Rev. E 89, 013204 (2014).

[17] J. Belmonte-Beitia, V. M. Pérez-García, V. Vekslerchik, and P. J. Torres, Phys. Rev. Lett. 98, 064102 (2007).

[18] G. Theocharis, P. Schmelcher, P. G. Kevrekidis, and D. J. Frantzeskakis, Phys. Rev. A 72, 033614 (2005).

[19] A. T. Avelar, D. Bazeia, and W. B. Cardoso, Phys. Rev. E 79, 025602(R) (2009).

[20] D.-S. Wang, X.-H. Hu, and W. M. Liu, Phys. Rev. A 82, 023612 (2010).

[21] B. J. Dabrowska-Wüster, E. A. Ostrovskaya, T. J. Alexander, and Y. S. Kivshar, Phys. Rev. A 75, 023617 (2007).

[22] R. M. Bradley, B. Deconinck, and J. N. Kutz, J. Phys. A: Math. Gen. 38, 1901 (2005).

[23] L. Li, B. A. Malomed, D. Mihalache and W. M. Liu, Phys. Rev. E 73, 066610 (2006).

[24] D. Yan, J. J. Chang, C. Hamner, P. G. Kevrekidis, P. Engels, V. Achilleos, D. J. Frantzeskakis, R. Carretero-Gonzalez, and P. Schmelcher, Phys. Rev. A 84, 053630 (2011).

[25] Z. H. Zhang, C. Zhang, S. J. Yang and S. P. Feng, J. Phys. B: At. Mol. Opt. Phys. 45, 215302 (2012).

[26] Z. H. Zhang, Y. K. Liu, and S. J. Yang, Mod. Phys. Lett. B 27, 1350013 (2013).

[27] B. Wu, J. Liu, and Q. Niu, Phys. Rev. Lett. 88, 034101 (2002). 
[28] L.D. Carr, C.W. Clark, and W.P. Reinhardt, Phys. Rev. A 62, 063610 (2000).

[29] L.D. Carr, C.W. Clark, and W.P. Reinhardt, Phys. Rev. A 62, 063611 (2000). 


\section{Figure Captions}

Figure 1 (a) The density profiles of the solution (6). $c_{0}=30, q=$ $-13.7707, m=0.4$ and $\mu=300$. (b) The density profiles for the single soliton solution (11). $c_{0}=1.3, k=2, q=-2$ and $\mu=5$.

Figure 2 (a) The density profiles of the solution (12) with $c_{0}=-10$, $q=22.734, m=0.5$ and $\mu=-200$. (b) The density profiles for the the single soliton solution corresponding to periodic solution (12) with $c_{0}=-1$, $k=3, q=4.5$ and $\mu=-1.5$.

Figure 3 The phase profile (a) and the density profile (b) for the complex solutions (16). The parameters are $n=2, m=0.82, q=17$, and $\mu=$ 448.6349. The phase profile (c) and the density profile (d) for the single soliton solution (22) with $n=6, k=8.7, q=5$, and $\mu=130.69$.

Figure 4 The phase (a) and the density (b) distributions of complex solutions (23) for the $F=1$ condensates with $n=2, m=0.44, q=-190, \mu=4$. (c) and (d) are the phase and density distributions for the single soliton solutions of (23) with $n=2, k=3, q=-8$, and $\mu=-20$.

Figure 5 The profiles of phase (a) and the density (b) for $F=2$ BEC solution (29). The parameters are $n=2, m=0.7, q=-53.4153, \mu=$ 210. The profiles of phase (c) and density (d) for the single soliton solution corresponding to (29) with $n=5, k=4.05, q=-8.2012$, and $\mu=200$. 
Fig.1

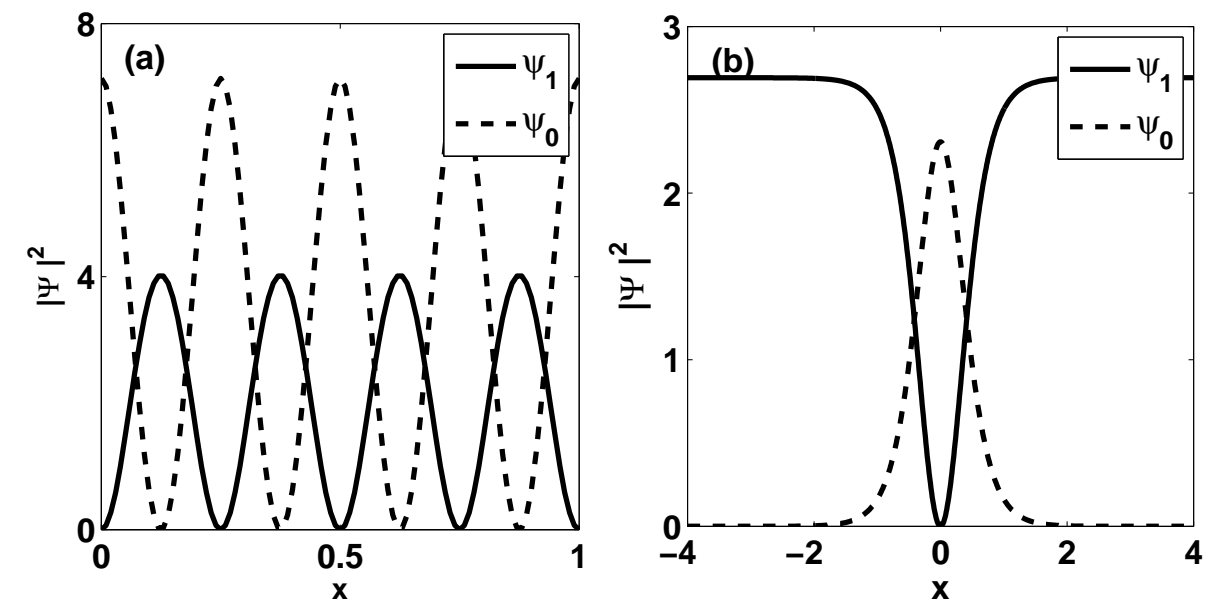


Fig.2

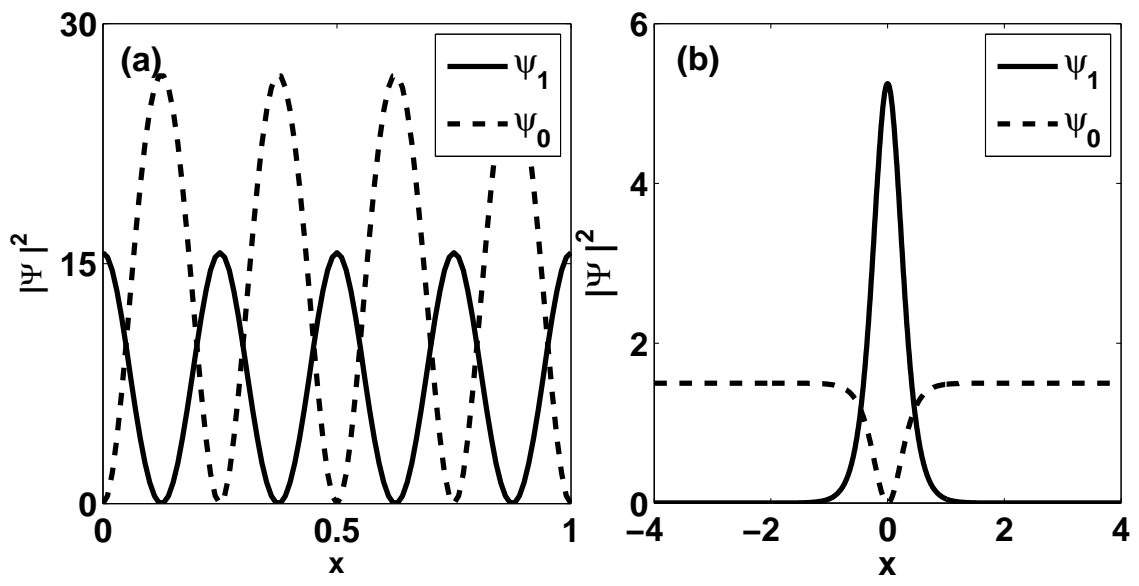


Fig.3
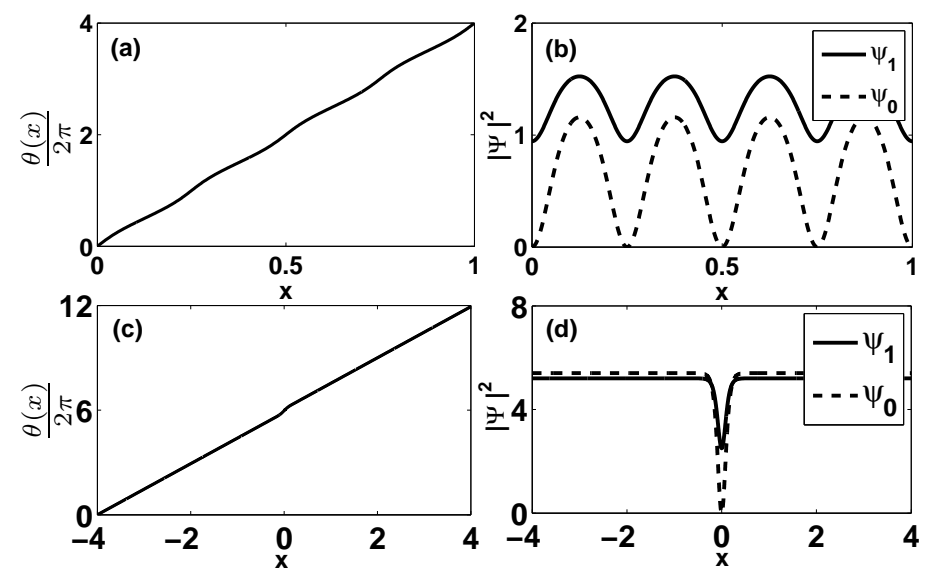
Fig.4
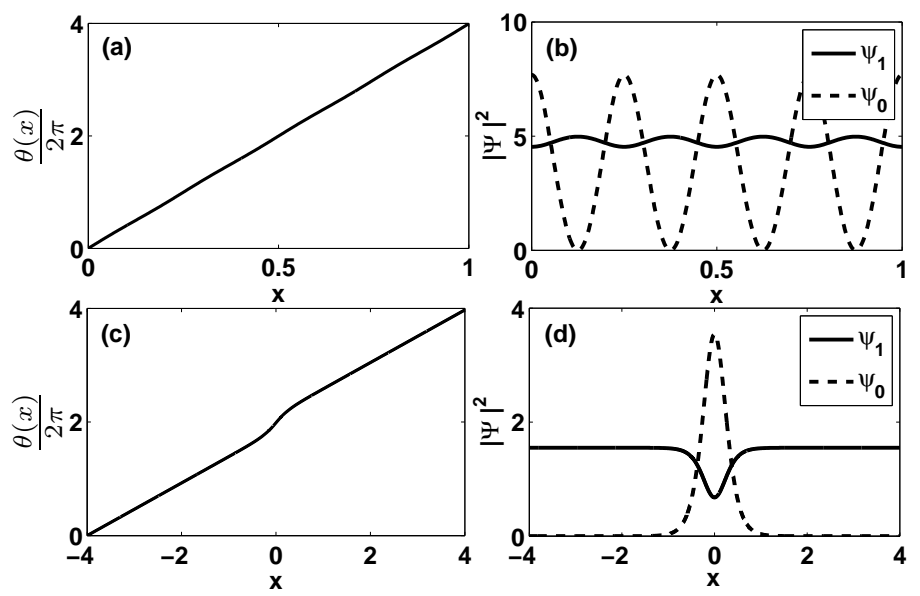
Fig.5
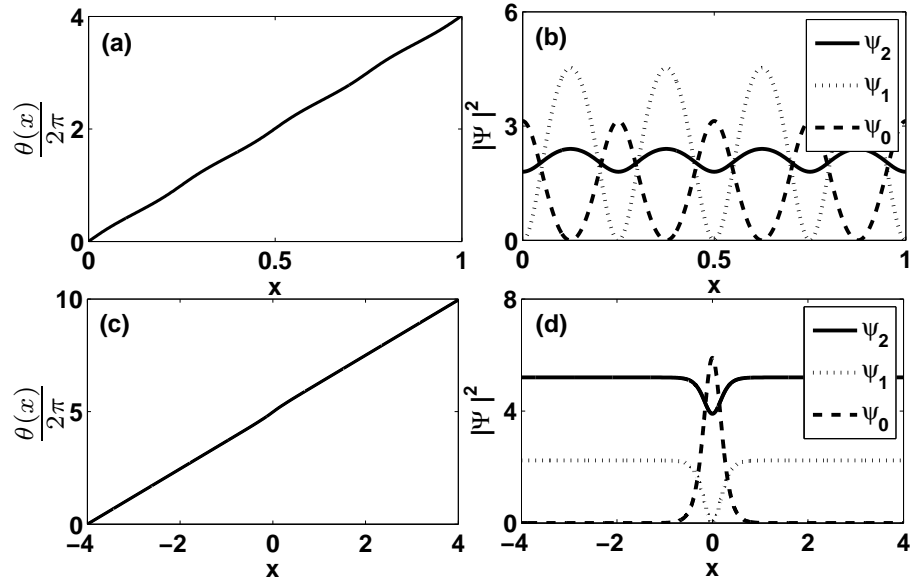